\title{
Comparison of the effects of joint mobilization, gym ball exercises, and breathing exercises on breathing pattern disorders and joint position sense in persons with chronic low back pain
}

\author{
Chae-Gil Lim \\ Department of Physical Therapy, College of Health Science, Gachon University, Incheon, Republic of Korea
}

Objective: To compare the effects of joint mobilization, gym ball exercises, and breathing exercises on breathing pattern disorders and joint position sense in persons with chronic lower back pain.

Design: Three-group pretest-posttest design.

Methods: Thirty-six individuals with chronic low back pain who were undergoing a postural correction and vertebral movement at a rehabilitation center participated in this study. The subjects were randomly divided into the joint mobilization group ( $\mathrm{n}=12$ ), gym ball exercises group $(n=12)$, and the breathing exercises group $(n=12)$. The exercises were applied for 40 minutes a day, twice a week for a total of 12 weeks. Measurement tools included the end-tidal $\mathrm{CO}_{2}\left(\mathrm{ETCO}_{2}\right)$, respiration rate (RR), breath hold time, Nijmegen Questionnaire (NQ), excursion, and joint position error (JPE).

Results: The groups showed significant differences in the $\mathrm{ETCO}_{2}, \mathrm{RR}, \mathrm{NQ}$, Excursion and JPE test before and after the intervention $(p<0.05)$. The differences between the groups were significant in the group that received the gym ball and breathing exercises in $\mathrm{ETCO}_{2}$ and $\mathrm{RR}(p<0.05)$. The differences between the groups were most significant in the group that received breathing exercises in NQ and excursion $(p<0.05)$. The differences between the groups were significant in the group that received the gym ball and breathing exercises in JPE Lt. and Rt. $(p<0.05)$.

Conclusions: All three interventions had a significant impact on the biomechanical changes, respiratory variables, and joint position sense in participants with chronic lower back pain. Breathing exercises were found to be particularly effective in improving respiratory parameters.

Key Words: Breathing exercises, Breathing pattern, Gym ball, Low back pain, Musculoskeletal manipulations, Proprioception sense

\section{Introduction}

Back pain is often a result of obesity, as well as psychological [1] and biomechanical factors [2] among other risk factors [3]. The magnitude of mechanical load on the spine is highly related to back pain [4], and excessive mechanical load on the lumbar spine can lead to intervertebral disc rupture or vertebral fracture. A strong association has been found between compression and shear force and the preva- lence of lumbar pain [5]. Thus, a comprehensive understanding of spinal biomechanics is important for the implementation of appropriate strategies to mitigate the risk of back pain while performing load-bearing tasks.

Mechanical receptors in joint tissues, articular capsules, and ligaments are stimulated by active and passive movement of the joints [6]. A pain response is induced when these joint tissues deform at the limits of normal tissue stretch. Pain is mediated by nociceptors, specialized peripheral sen-

Received: 28 February, 2020 Revised: 11 March, 2020 Accepted: 12 March, 2020

Corresponding author: Chae-Gil Lim (ORCID https://orcid.org/0000-0001-9508-9455)

Department of Physical Therapy, College of Health Science, Gachon University, 191 Hambangmoe-ro, Yeonsu-gu, Incheon 21936, Republic of Korea Tel: 82-32-820-4424 Fax: 82-32-820-4069 E-mail: jgyim@gachon.ac.kr

(c) This is an Open-Access article distributed under the terms of the Creative Commons Attribution Non-Commercial License (http://creativecommons.org/licenses/ by-nc/4.0) which permits unrestricted non-commercial use, distribution, and reproduction in any medium, provided the original work is properly cited.

Copyright $(\odot 2020$ Korean Academy of Physical Therapy Rehabilitation Science 
sory neurons that convert these stimuli into electrical signals, which are relayed to higher brain centers. Stimulation of nociceptors is potentially damaging [7], and if noxious stimuli persist, peripheral and central sensitization may progress, causing pain to transition from acute to chronic.

Breathing pattern can affect the body and consciousness, and breathing pattern abnormalities may cause mechanical, physiological, and psychological dysfunction [8]. In individuals with chronic lower back pain, there was decreased muscle movement in the front and middle portion of the diaphragm during isometric contractions of the upper and lower limbs, and this muscle recruitment, along with steep changes in the middle and rear parts of the diaphragm, is likely to cause sprains in the posterior region of the spine. If the diaphragm is abnormally contracted and activated, it may cause chronic lower back pain and movement dysfunction [9].

Chaitow et al. [10] reported abnormal posture as a major cause of breathing pattern disorder (BPD), with the physiological and anatomical aspects of respiration being interconnected. When breathing pattern abnormalities occur, accessory respiratory muscles and the diaphragm do not return to a stable position after shortening, causing unstable exhalation. Excessive lung swelling and pressure result in accelerated respiratory distress [11]. The BPD can be caused by repeated exercise learning. When relaxed, excessive contraction of the oblique muscles act as a corset to prevent the expansion of the diaphragm, causing changes in the breathing pattern of the upper chest [12]. A large number of individuals with chronic pain caused by musculoskeletal disorders reported having respiratory pattern abnormalities resulting in large upper pectoral movements without respiratory problems [13]. Stable contraction of the diaphragm increases pressure and provides stability to the lumbar spine and torso [14], while inadequate diaphragm coordination has been reported to cause lumbar spine instability, motor control changes, and movement dysfunction [15]. Previous studies have reported abnormal breathing patterns in persons with chronic lower back pain $[9,13]$, and those with chronic pain are slow to adapt proprioceptively to environmental changes due to impaired joint position sense transmission [16].

Joint position sense, a specific type of proprioception, is the ability to perceive the position of a joint without external cues and is mediated by sensory information transmitted to the central nervous system by mechanical receptors converting cues to electrical signals in nerve endings [17,18]. Joint position error (JPE) can be measured by examining the abil- ity of a joint to return to its initial position after spontaneous movement [19]. Cervical JPE is assessed after spontaneous movement in situ via cervical extension, flexion, and rotation [19]. Deviations from normal function in these tests may result from altered afferent neuronal input in the neck [20]. In persons with chronic lower back pain, JPE symptoms are associated with respiratory dysfunction, which shortens the respiratory muscles, such as the sternocleidomastoid, trapezius, and scalene muscles, that cause movement in the cervical spine. Because respiratory pattern error affects motor control of the shoulder and the lumbar and cervical spine, it impedes functional movement in which diaphragmatic breathing plays an important role [21]. Therefore, in the treatment of those with chronic lower back pain, the restoration of biomechanical spinal movement, recovery of a normal breathing pattern, and restoration of joint position sense are thought to be necessary for overall functional recovery.

A lumbar joint manipulation technique [22] and lumbar pelvic stabilization exercises $[23,24]$ are used to improve lumbar spine and pelvic function in individuals with chronic lower back pain. Diaphragmatic breathing exercises [25] and respiratory exercises using auditory and tactile feedback [26] have been reported to improve motor control and respiratory function [27]. In particular, gym ball exercises have been frequently used to improve pelvic and spinal stability and assist with proprioception and postural control in persons with chronic lower back pain by activating the local muscles [28]. Furthermore, Sremakaew et al. [29] found that cervical spine mobilization and stabilization and proprioception exercises improved afferent sensory input and reduced pain; however, the authors did not fully explore the effects of these treatment approaches on improved outcomes. In clinical practice, stabilization exercises and manual therapy are primarily used for treating chronic lower back pain [30]. Also, breathing exercise programs were shown to be effective in improving lung function, reducing back pain, and improving quality of life [5]. Nevertheless, breathing exercises are used only as an adjuvant, and evidence is insufficient to determine whether they are suitable as an intervention.

The purpose of this study was to investigate the effects of joint mobilization, gym ball exercises, and breathing exercises on the biomechanical changes, respiratory patterns, and joint position sense in persons with chronic lower back pain. 


\section{Methods}

\section{Participants}

This study was a two-arm, parallel, randomized controlled trial with concealed allocation and researcher and assistant blinding. Between November 2018 and April 2019, a total of 45 patients were admitted to the rehabilitation center, and 42 fulfilled the inclusion criteria. Participants were randomly assigned to the joint mobilization group (JMG) (n= $14)$, the gym ball exercises group (GBG) $(n=13)$, or the breathing exercises group (BEG) $(n=15)$. Thirty-six participants completed the study (Figure 1). Individuals diagnosed with chronic lower back pain 12 months after symptom onset at the rehabilitation center were included in this study. The inclusion criteria were as follows: (1) those with nonspecific chronic lower back pain and mechanical chronic lower back pain, (2) those using thoracic breathing method, and (3) those who understood and agreed to the contents of this study. In regards to the exclusion criteria, those with the following were excluded from the study: (1) previous orthopedic and neurosurgical surgery, (2) cardiovascular disease and high risk of falls, (3) other chronic pain, (4) participation in other exercise programs (abdominal muscle training within 1 year), (5) pregnancy within 2 years prior, (6) malignant tumor, and (7) radiating pain at two sites [31]. All subjects understood the requirements of the study and provided informed consent before their participation. All procedures were confirmed by the institutional review board of the institution and the Helsinki Declaration was followed.

\section{Experimental procedure}

Preliminary examinations were performed to document the general characteristics of each subject, such as sex, age, weight, and height. Scores on the modified Visual Analogue

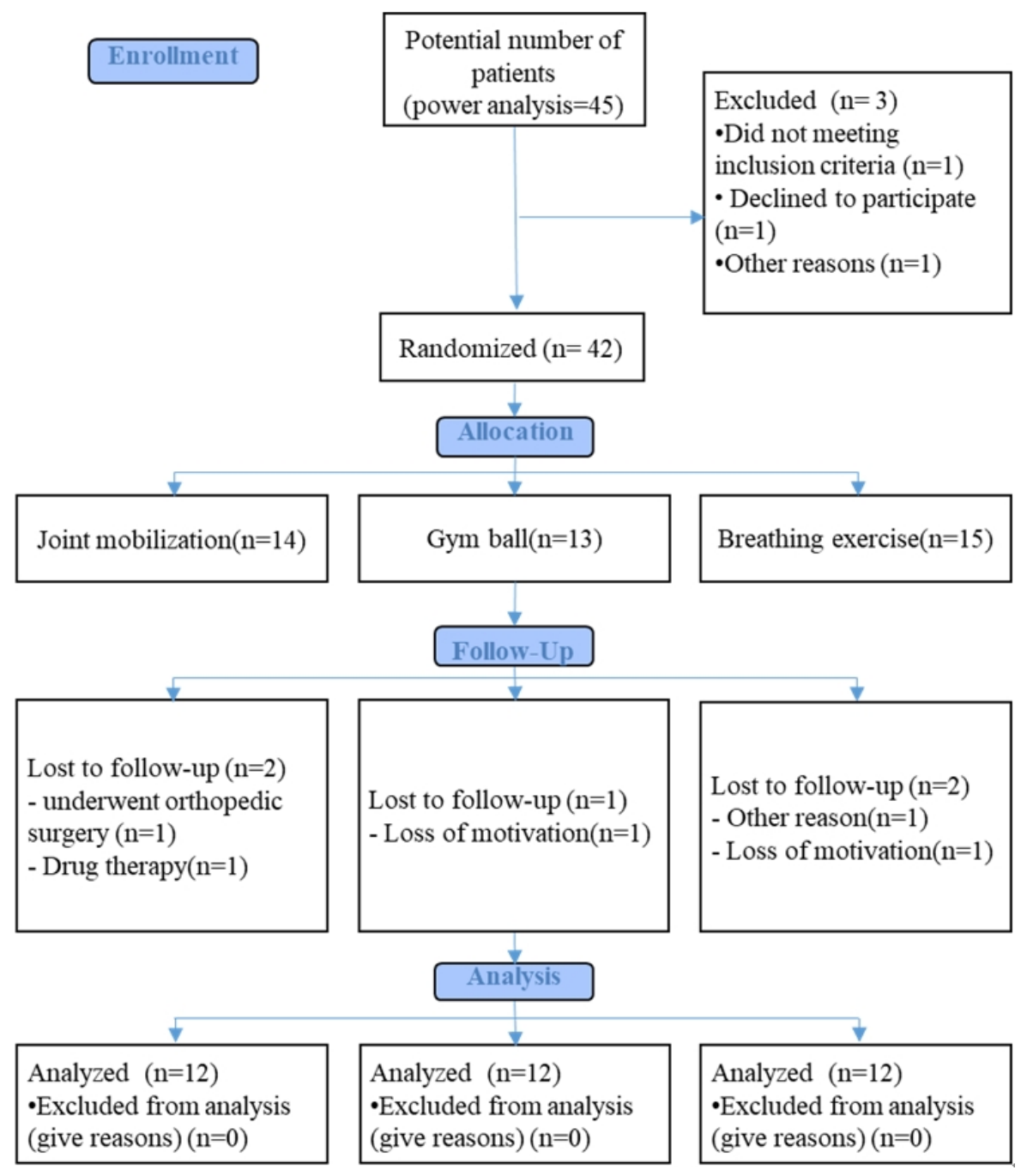

Figure 1. Flow diagram of this study. Forty-two individuals were enrolled in the study and were randomly assigned to the joint mobilization group ( $\mathrm{n}=14)$, the gym ball group $(n=13)$ or the breathing exercise group $(n=15)$. 
Scale and the Nijmegen Questionnaire (NQ) were recorded before and after the experiment.

Capnographs were used for biochemical examination of respiratory function [32]. Subjects were seated in a comfortable position with a nasal cannula inserted, and end-tidal carbon dioxide $\left(\mathrm{ETCO}_{2}\right)$ and respiratory rate $(\mathrm{RR})$ were measured. After removing the nasal cannula, breath hold time (BHT) [33] and diaphragmatic breathing were assessed using the Hi-Lo method [34]. After respiratory function evaluation, a five minutes rest period was allowed, and the joint position sense of the cervical spine was assessed.

For the assessment of cervical joint position sense, subjects were instructed to be seated comfortably on the ground with the head in a neutral position and to direct a laser pointer attached to a headset at a target in front of them. The subjects performed three movements (neck extension, right rotation, and left rotation) and returned the laser pointer to the starting position after each. The subjects were allowed to practice each movement once. In the actual experiment, the subject had their eyes closed, performed the movement up to the maximum possible range, and returned to the starting position. Each movement was measured three times. After each measurement, the researcher manually returned the laser pointer to its original position. Between different movements, the subjects were allowed to open their eyes and correct their starting point before they were blindfolded again. Cervical JPE was based on the average of the absolute error values for neck extension, right rotation, and left rotation.

\section{Outcome measures}

\section{Capnography}

Capnography (YM6000, Mediana Co., Wonju, Korea) was used to measure the $\mathrm{ETCO}_{2}$ and RR (Figure 2A). $\mathrm{ETCO}_{2}$ less than $35 \mathrm{mmHg}$, RR of 16 breaths/min or more,
BHT of less than 20 seconds, and an NQ score of over 23 were considered as signs of respiratory pattern abnormality $[21,34,35]$.

\section{Joint position error test}

A cervical JPE test was used as an afferent input disorder test of the cervical spine [36]. A small laser pointer was attached to the headset with the subject sitting and looking at a wall in the front $90 \mathrm{~cm}$ away from the laser-projected point, with the eyes closed and active neck movement. After performing a movement, subjects attempted to return to the starting point. The final laser pointer position was measured with respect to the starting point in $\mathrm{cm}$. A deviation of $4.5^{\circ}$ or less from the center of the target was considered normal. At a distance of $90 \mathrm{~cm}$ from the center of the target, a $4.5^{\circ}$ deviation corresponded to $7 \mathrm{~cm}$. This method was used as a quantitative evaluation tool for measuring abnormal positional sense of the cervical joint with an error of $3^{\circ}-4^{\circ}$. The error was measured for each subject after active cervical spine extension and rotation (Figure 2B) [19]. This is a validated test method with a high test-retest reliability [37].

\section{Excursion test}

In order to determine the extent to which the thoracic cage expanded during breathing, the circumference of the thoracic cage was measured using a tape measure [38]. We measured the thoracic circumference of the subject standing upright during breathing by placing a tape measure around the chest at the level of the xiphoid process in the front and the 10th thoracic vertebral segment in the back. Chest motility was recorded as the mean value of the difference between chest circumferences during maximum exhalation and maximum inhalation [39].
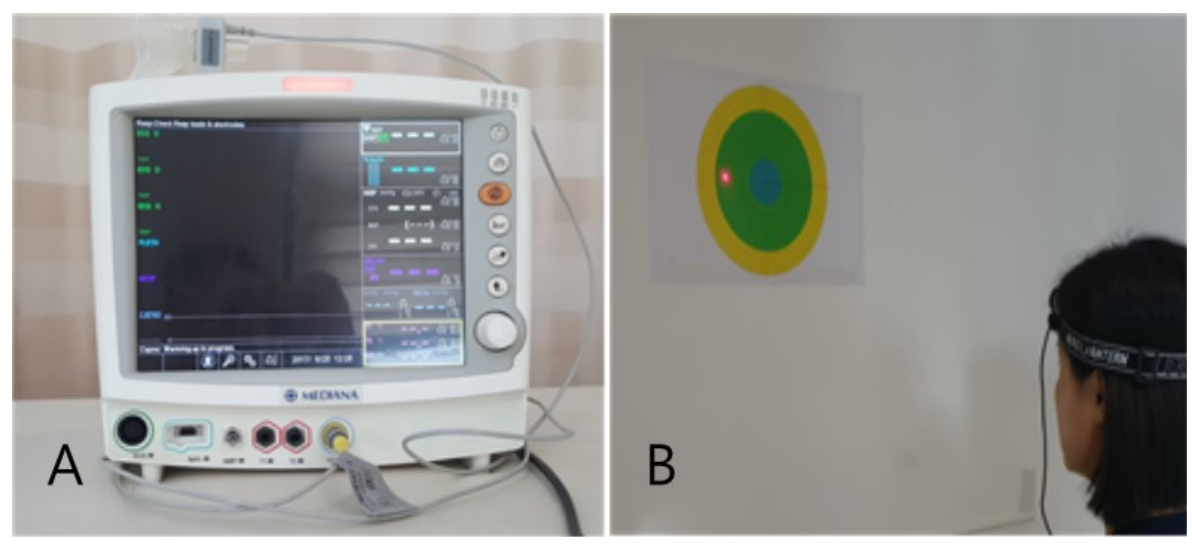

Figure 2. (A) Capnography (YM6000, Mediana Co., Wonju, Korea). (B) Joint position error test. 


\section{Intervention procedures}

\section{Joint mobilization}

Joint mobilization was divided into three stages for recovery of motion and reduction of pain. First the joint at the treatment site was slacked, then the slack was taken up, and finally the joint was stretched. Stages 1 and 2 were used to alleviate pain, and stage 3 was used to increase mobility. Initiation of treatment was followed by determining the resting or actual resting positions followed by traction and slipping. When both muscles and joints were found to be abnormal, general massage was applied to the muscles and functional massage was then applied to both the muscles and joints before joint mobilization treatment was initiated [40,41]. The subjects underwent joint mobilization for 40 minutes, twice a week, for 12 weeks by a physical therapist who had attended a Kaltenborn orthopedic physiotherapy spine course.

\section{Gym ball exercises}

During the first six weeks of exercise, subjects performed side bridging, gym ball partial-curl ups, supine bridging with single leg raise, prone bridge, and quadruped exercise with relatively stable support. Subjects performed gym ball push-ups, gym ball single leg holds, and gym ball roll-outs with an unstable support for six weeks. Gym ball exercises were applied to improve lumbar spine stability and increase abdominal activity $[42,43]$. The subjects underwent gym ball exercise for 40 minutes, twice a week, for 12 weeks overseen by a physical therapist.

\section{Breathing exercises}

The respiratory exercise regimen was designed to maintain neutral alignment of the spine by retraining the breathing pattern through relaxed diaphragm breathing. The first method used was the doming technique, which relaxes the diaphragm during rest and improves diaphragm contraction and relaxation functions $[43,44]$. The subject was seated at a treatment table in a comfortable and relaxed position. The therapist wrapped his arms around the subject's rib cage from behind with hands under the ribs. Then, the therapist carefully turned the torso of the subject to the left and right sides so that the rib cage moved freely and comfortably while the subject attempted to relax the diaphragm and improve ease of movement. This posture was held for $5 \mathrm{mi}-$ nutes (Figure 3). The second method involved training and correcting the breathing patterns and involved several steps:

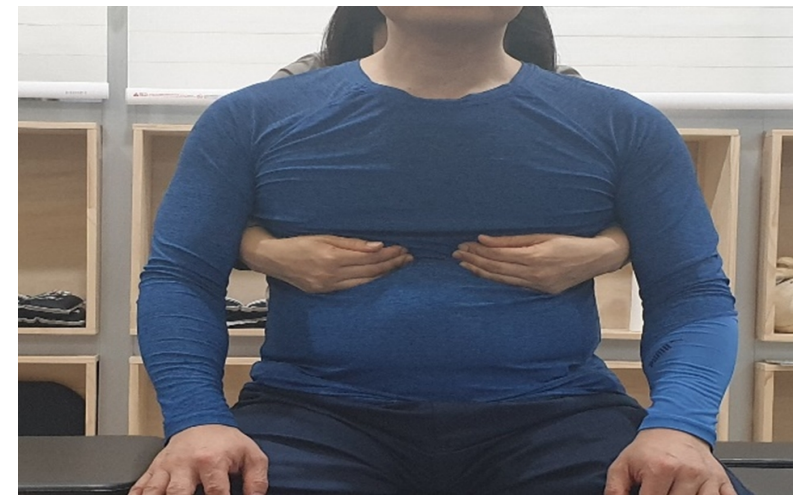

Figure 3. Doming technique of the diaphragm.

(1) recognizing abnormal breathing patterns, (2) relaxing the jaws, upper chest, shoulders, and accessary respiratory muscles, (3) retraining abdominal/diaphragm breathing patterns including normal breathing frequency and rhythm and talking at rest, (4) training awareness of RR and rhythm during activity [10]. Third, subjects were educated on normal breathing patterns in functional movements corresponding to various postures and movements performed in daily life [45]. The subjects underwent breathing exercises for $40 \mathrm{mi}-$ nutes, twice a week, for 12 weeks overseen by a physical therapist [43].

\section{Data analysis}

To verify normal distribution of the data, all data were tested using the Kolmogorov-Smirnov test. Descriptive statistical analysis and ANOVA were used to assess the general characteristics and test for homogeneity among the subjects. In order to analyze differences between the dependent variables according to the measurement period (before and after the experiment) and the intervention method (3 types), multivariate ANOVA was performed and statistically significant differences were identified using Tukey's HSD. The significance level was set at $\alpha=0.05$. All data were analyzed using IBM SPSS Statistics for Windows, Version 25.0 (IBM Co., Armonk, NY, USA).

\section{Results}

General baseline characteristics are shown in Table 1.

\section{Comparison of breathings pattern disorder}

A comparison of the $\mathrm{ETCO}_{2}, \mathrm{RR}, \mathrm{BHT}, \mathrm{NQ}$, and excursion scores before and after intervention among the three groups is presented in Table 2. Significant differences in 
Table 1. General characteristics of the subjects

$(\mathrm{N}=36)$

\begin{tabular}{lccrr}
\hline \multicolumn{1}{c}{ Variable } & Joint mobilization $(\mathrm{n}=12)$ & Gym ball exercises $(\mathrm{n}=12)$ & Breathing exercises $(\mathrm{n}=12)$ & $\mathrm{F}(p)$ \\
\hline Sex (male/female) & $4 / 8$ & $6 / 6$ & $4 / 8$ & $0.44(0.648)$ \\
Age (y) & $45.33(10.27)$ & $41.00(6.38)$ & $43.33(10.26)$ & $0.67(0.517)$ \\
Height (cm) & $165.56(6.28)$ & $166.20(8.72)$ & $165.44(6.95)$ & $0.03(0.964)$ \\
Weight (kg) & $63.00(7.16)$ & $65.80(7.84)$ & $63.25(7.01)$ & $0.53(0.591)$ \\
BMI (score) & $22.90(1.24)$ & $23.76(1.46)$ & $23.01(1.80)$ & $1.13(0.332)$ \\
VAS (score) & $4.91(1.08)$ & $5.66(1.30)$ & $5.00(1.04)$ & $0.62(0.538)$ \\
\hline
\end{tabular}

Values are presented as number only or mean (SD).

BMI: body mass index, VAS: Visual Analogue Scale.

Table 2. Comparison of before and after intragroup intervention on breathing pattern disorder $(\mathrm{N}=36)$

\begin{tabular}{lccc}
\hline Variable & Pre & Post & $\mathrm{t}(p)$ \\
\hline ETCO $_{2}(\mathrm{mmHg})$ & & & \\
JMG & $30.91(2.77)$ & $31.83(1.64)$ & $-1.30(0.218)$ \\
GBG & $31.25(2.80)$ & $34.41(2.60)$ & $-3.64(0.004)$ \\
BEG & $31.08(3.11)$ & $37.16(1.69)$ & $-7.17(<0.001)$ \\
RR (breaths/min) & & & \\
JMG & $17.58(3.84)$ & $17.00(2.44)$ & $0.42(0.679)$ \\
GBG & $18.16(4.80)$ & $17.50(4.29)$ & $2.96(0.013)$ \\
BEG & $21.00(4.69)$ & $15.08(3.08)$ & $2.95(0.013)$ \\
BHT (sec) & & & \\
JMG & $36.25(7.30)$ & $39.66(5.49)$ & $-3.40(0.006)$ \\
GBG & $42.75(9.71)$ & $46.58(15.25)$ & $-1.35(0.204)$ \\
BEG & $40.16(9.11)$ & $50.83(11.02)$ & $-2.77(0.018)$ \\
NQ (score) & & & \\
JMG & $22.42(10.73)$ & $18.17(8.76)$ & $2.66(0.022)$ \\
GBG & $22.25(7.62)$ & $21.08(6.85)$ & $2.88(0.015)$ \\
BEG & $22.67(8.79)$ & $13.17(4.70)$ & $5.96(<0.001)$ \\
Excursion (cm) & & & \\
JMG & $2.70(0.73)$ & $4.25(0.87)$ & $-3.84(0.003)$ \\
GBG & $2.09(0.90)$ & $4.83(0.81)$ & $-9.58(<0.001)$ \\
BEG & $2.22(1.05)$ & $6.06(0.82)$ & $-7.91(<0.001)$ \\
\hline
\end{tabular}

Values are presented as mean (SD).

$\mathrm{ETCO}_{2}$ : end-tidal carbon dioxide, JMG: joint mobilization group, GBG: gym ball group, BEG: breathing exercise group, RR: respiration rate, BHT: breath holding time, NQ: Nijmegen Questionnaire.

$\mathrm{ETCO}_{2}$ and RR scores before and after intervention were found in the GBG and BEG $(p<0.05)$. Significant differences in BHT scores before and after intervention were found in the JMG and BEG $(p<0.05)$. Significant differences in NQ and excursion scores before and after intervention were found in the JMG, GBG, and BEG $(p<0.05)$. A comparison between the three groups before and after intervention is presented in Table 3. Significant differences in the $\mathrm{ETCO}_{2}, \mathrm{RR}$, NQ, and excursion scores after intervention were found between the groups $(p<0.05)$. Post-hoc tests revealed that the BEG was significantly different from the $\mathrm{JMG}$ and $\mathrm{GBG}$ in the $\mathrm{ETCO}_{2}, \mathrm{RR}, \mathrm{NQ}$, and excursion scores $(p<0.05)$. The BHT scores were not significantly different between the JMG, GBG, and BEG $(p>0.05)$.

\section{Comparison of joint position error}

A comparison of the JPE left rotation (JPE_Lt. R), JPE right rotation (JPE_Rt. R), and JPE extension (JPE_EXT) scores before and after intervention among the three groups is presented in Table 4. Significant differences in the JPE Lt. R, JPE_Rt. R, and JPE_EXT scores before and after intervention were found in the BEG $(p<0.05)$. Significant differences in JPE_Rt. R scores before and after intervention were found in the GBG $(p<0.05)$. Significant differences in NQ and excursion scores before and after intervention were found in the JMG, GBG, and BEG $(p<0.05)$. A comparison between the three groups before and after intervention is presented in Table 5. Significant differences in the JPE_Lt. R, JPE_Rt. R scores after intervention were found between the groups $(p<0.05)$. Post-hoc tests revealed that the GBG and BEG was significantly different from the JMG in the JPE_Lt. R, JPE_Rt. R scores $(p<0.05)$. The JPE_EXT scores were not significantly different between the JMG, GBG, and $\operatorname{BEG}(p>0.05)$.

\section{Discussion}

This study compared the biomechanical changes, breathing pattern, and joint positional sense in persons with chronic lower back pain. Forty-two participants with chronic lower back pain were randomly divided into 3 groups: joint manipulation group, GBG, and BEG. Thirty-six participants completed the study.

Bradley and Esformes [21] reported that the normal range 
Table 3. Comparison of intergroup intervention on breathing pattern disorder

$(\mathrm{N}=36)$

\begin{tabular}{lrrrr}
\hline \multicolumn{1}{c}{ Variable } & JMG $(\mathrm{n}=12)$ & GBG $(\mathrm{n}=12)$ & BEG $(\mathrm{n}=12)$ & $\mathrm{F}(p)$ \\
\hline $\mathrm{ETCO}_{2}(\mathrm{mmHg})$ & $-0.91(2.42)$ & $-3.16(3.01)^{*}$ & $-6.08(2.93)^{\dagger}$ & $10.24(<0.001)$ \\
$\mathrm{RR}($ breaths/min) & $0.58(4.75)$ & $0.66(0.77)^{*}$ & $5.91(6.94)^{*}$ & $4.70(0.016)$ \\
$\mathrm{BHT}(\mathrm{sec})$ & $-3.41(3.47)$ & $-3.83(9.83)$ & $-10.66(13.33)$ & $2.08(0.141)$ \\
$\mathrm{NQ}(\mathrm{score})$ & $4.25(5.52)$ & $1.16(1.40)$ & $9.50(5.51)^{*}$ & $10.14(<0.001)$ \\
Excursion $(\mathrm{cm})$ & $-1.50(1.35)$ & $-2.74(0.99)$ & $-3.84(1.68)^{*}$ & $8.76(0.001)$ \\
\hline
\end{tabular}

Values are presented as mean (SD).

JMG: joint mobilization group, GBG: gym ball group, BEG: breathing exercise group, $\mathrm{ETCO}_{2}$ : end-tidal carbon dioxide, RR: respiration rate, BHT: breath holding time, NQ: Nijmegen Questionnaire.

*Significant difference in a comparison with the JMG $(p<0.05)$. ${ }^{\dagger}$ Significant difference in a comparison with the GBG $(p<0.05)$.

Table 4. Comparison of before and after intragroup intervention on JPE

\begin{tabular}{lrrr}
\hline Variable & Pre & \multicolumn{1}{c}{ Post } & $\mathrm{t}(p)$ \\
\hline JPE_Lt. R (cm) & & & \\
JMG & $11.46(2.97)$ & $11.50(2.50)$ & $-0.14(0.888)$ \\
GBG & $10.79(2.59)$ & $8.60(1.96)$ & $2.08(0.061)$ \\
BEG & $11.35(2.47)$ & $6.17(1.92)$ & $7.80(0.001)$ \\
JPE_Rt. R (cm) & & & \\
JMG & $10.78(2.54)$ & $10.87(2.50)$ & $-0.33(0.744)$ \\
GBG & $10.38(2.20)$ & $6.84(2.79)$ & $2.96(0.013)$ \\
BEG & $12.40(3.06)$ & $6.26(2.74)$ & $5.69(0.001)$ \\
JPE_EXT (cm) & & & \\
JMG & $8.83(2.10)$ & $7.85(2.71)$ & $1.09(0.299)$ \\
GBG & $10.06(2.19)$ & $7.87(2.70)$ & $2.11(0.058)$ \\
BEG & $9.34(2.78)$ & $5.01(1.70)$ & $4.77(0.001)$ \\
\hline
\end{tabular}

Values are presented as mean (SD).

JPE: joint position error, JMG: joint mobilization group, GBG: gym ball group, BEG: breathing exercise group, JPE_Lt. R: JPE left rotation, JPE Rt. R: JPE right rotation, JPE EXT: JPE extension.

of $\mathrm{ETCO}_{2}$ is $35-40 \mathrm{mmHg}$, while $\mathrm{ETCO}_{2}$ of less than 35 $\mathrm{mmHg}$ is considered to indicate a BPD. Chest breathing can have a serious effect on the chemical aspects of breathing. A decrease in bloodstream $\mathrm{CO}_{2}$ level leads to an increase in blood $\mathrm{pH}$ and results in respiratory alkalosis [46,47], which can cause changes in physiological, psychological and neurological conditions and negatively affect the musculoskeletal system [8]. The diaphragm, the main breathing muscle, plays an essential role in controlling the spine during postural control [48]. Increasing demand for diaphragmatic respiration inhibits the diaphragmatic contribution to torso stabilization [49]. The breathing exercises in this study focused on improving diaphragm function and restoring normal breathing and postural control. Significantly higher $\mathrm{ETCO}_{2}$ levels and respiratory movement in the BEG than in the JMG are likely the result of the activation of suppressed diaphragm movement in subjects with chronic lower back pain, resulting in a change in breathing pattern from chest breathing to diaphragmatic breathing.

Respiratory dysfunction or respiratory failure is a chronic or repetitive change in the respiratory pattern that contributes to respiratory and non-respiratory discomfort [50]. Symptoms of BPD include dyspnea with normal lung function, chest tightness, chest and musculoskeletal pain, deep sighs, shortness of breath during exercise, frequent yawning, and hyperventilation [51]. Hyperventilation is a condition in which respiration occurs above the normal metabolic rate, leading to a sharp decrease in the partial pressure of carbon dioxide $\left(\mathrm{PaCO}_{2}\right)$ and predictable physiological changes [51]. A sharp decrease in $\mathrm{PaCO}_{2}$ in the arteries and tissues leads to more frequent breathing to increase oxygen supply. ARR of more than 16 breaths per minute is regarded as dysfunctional, and hyperventilation increases the RR, which leads to biochemical changes in the human body [34].

The breathing exercise group showed a normal RR after the intervention. The breathing exercises aimed to alter the breathing pattern from chest breathing to diaphragmatic breathing allows sufficient oxygen supply to the tissues. This can be inferred from the fact that abnormal over-breathing was eliminated and the RR was lowered. Breathing exercises showed a more significant effect on RR than joint mobilization or gym ball exercises.

Breath holding time is an aspect of respiratory function and is generally disturbed in people with respiratory disabilities [52]. Stark and Stark [53] suggested that a BHT of less than 20 seconds is indicative of an abnormal breathing pattern, which also correlates with $\mathrm{ETCO}_{2}$ levels. However, other studies showed contradicting results [21]. In this study, the BHT of individuals with chronic lower back pain was greater than 20 seconds, suggesting that the sensitivity of this variable to indicate BPD is low. Nevertheless, BHT was greater in the breathing exercise group than in the other two 
Table 5. Comparison of intergroup intervention on JPE $(\mathrm{N}=36)$

\begin{tabular}{ccccc}
\hline Variable & JMG $(\mathrm{n}=12)$ & GBG $(\mathrm{n}=12)$ & BEG $(\mathrm{n}=12)$ & $\mathrm{F}(p)$ \\
\hline JPE_Lt. R & $-0.04(1.00)$ & $2.19(3.64)^{*}$ & $5.17(2.29)^{*}$ & $12.60(<0.001)$ \\
JPE_Rt. R & $-0.09(0.94)$ & $3.54(4.14)^{*}$ & $6.13(3.73)^{*}$ & $11.00(<0.001)$ \\
JPE_EXT & $0.97(3.09)$ & $2.19(3.59)$ & $4.32(3.13)$ & $3.20(0.054)$ \\
\hline
\end{tabular}

Values are presented as mean (SD).

JPE: joint position error, JMG: joint mobilization group, GBG: gym ball group, BEG: breathing exercise group, JPE_Lt. R: JPE left rotation, JPE_Rt. R: JPE right rotation, JPE_EXT: JPE extension.

*Significant difference in comparison with the JMG $(p<0.05)$.

groups. Although breathing exercises at normal levels were employed, this intervention was found to have an effect on respiratory parameters.

There was a statistically significant difference in the NQ scores among the three groups. Further, there was a statistically significant difference between before and after the intervention. The NQ was originally designed to assess one form of dysfunctional breathing (hyperpnea syndrome), and the symptoms identified by Nijmegen are mainly due to the presence of abnormal breathing patterns rather than hypocarbonism [33]. The NQ includes three symptoms, mainly related to the respiratory, peripheral neurovascular, or central nervous systems, and consists of four aspects including symptoms related to tension [54]. A score of 23 or more is considered to be indicative of an abnormal breathing pattern [21]. In this study, none of the three groups exceeded a score of 23 in the initial evaluation. After the intervention, the breathing exercise group scored lower than the other two groups. Although the breathing exercises in this study improved the symptoms included in the NQ, the effects on the symptoms related to the peripheral neurovascular and central nervous systems require further study.

Weakness of the respiratory muscles and limited expansion of the thorax may cause abnormalities in respiratory function, reducing the mobility and activity of the diaphragm [55]. Improved chest movements during respiration may indicate increased diaphragmatic activity and mobility. The most commonly used respiratory training methods during rehabilitation can improve the mobility of the thoracic cage by smoothly contracting the diaphragm, which is the working muscle for inhalation [25]. The breathing exercises performed in this study aimed to improve respiratory function and posture by relaxing the diaphragm and correcting abnormal breathing patterns. The BEG had significantly higher excursion after the intervention than the JMG or the GBG, indicating that the breathing exercises had a greater effect on this respiratory variable than the other methods. It is also likely that the breathing pattern changed to diaphragmatic breathing, which facilitates inhalation and exhalation due to improved movement and function of the diaphragm. More research is needed to clarify how the diaphragm moves and functions in conjunction with the chest.

Abnormal proprioception has been found to be associated with pain, exudation, trauma, fatigue, and a number of musculoskeletal disorders [56]. Proprioception of individuals with chronic lower back pain can be disturbed due to altered reflex activity and changes in the intensity of the gammamuscle spindle system through the Type III and IV afferent pain receptors [57].

In this study, we attempted to study the changes in joint position sense in the neck. There was a statistically significant difference between JPE_Lt. R, JPE_Rt. R among the groups, with these parameters showing significant improve after the intervention. Multiple comparisons between the intervention methods showed that JPE_Lt. R, JPE_Rt. R was significantly improved in the breathing exercise. Kolar et al. [9] reported altered breathing patterns in persons with chronic lower back pain. Chest breathing is often controlled by the accessary respiratory muscles (sternocleidomastoid, trapezius, and scalene muscles), which act predominantly in the lower thorax and abdomen [34]. Continuous use of the accessary respiratory muscles can cause muscle tension and neck pain [13]. Persistent chest breathing due to abnormal breathing patterns in those with chronic lower back pain may be related to neck pain, fatigue, accessary respiratory muscle spasm, and reduced proprioception in the neck. In this study, joint position sense improved after the performance of breathing exercises, which were successful in changing chest breathing to diaphragmatic breathing, resulting in decreased accessary muscle tension, fatigue, and pain. This improvement in proprioception may be due to the resolution of factors that interfere with it. This study confirmed that joint position sense can be affected by abnormal breathing patterns because breathing exercises were successful in cor- 
recting the breathing pattern while simultaneously improving proprioception. Also, joint mobilization can elongate stiffened tissue, increase joint motion range, facilitate normal movement of damaged joints, and prevent the worsening of symptoms. Proprioception through joint movement precedes harmful stimulus recognition and can stimulate normal nerve sensation to inhibit pain perception [58]. Stimulating the mechanical receptors of the joints using joint mobilization can inhibit harmful stimulation at the spinal cord or brainstem level. Santilli et al. [59] found that joint mobilization can be useful for treating disk and nerve damage. In this study, joint mobilization was used to decrease pain due to improved joint motion range and for restoration of normal mobility. Mechanical stimuli that move the joints have been found to inhibit harmful stimuli transmitted to the spinal cord or brainstem. Type I and II mechanoreceptors can be stimulated by joint mobilization over the range of motion of the joint. Mechanoreceptor stimulation activates large nerve fibers in the spinal cord that suppress input from small nerve fibers at the synapse, which in turn reduces pain awareness [60]. Thus, suppression of the synapses of type III and IV pain receptors in the ligaments via arthroplasty reduces the perception of pain.

Local muscles assist to control proprioception and posture, reducing the risk of trauma [61]. Gym ball exercises in this study were intended to simultaneously stimulate large muscles and local muscles. This was achieved by maintaining a neutral posture during the exercises. Local muscles contribute to stiffness maintenance and stability along the spinal column and are composed of a high percentage of type 1 muscle fibers, making them suitable for improving thoracic endurance. Gym ball exercises, which focus on maintaining a neutral spine, can improve thoracic endurance because the actions involved in such exercises are primarily performed by small muscles. Maintaining the spine position for as long as possible during these exercises can improve trunk endurance and reduce the potential for back pain [62]. Therefore, use of large and small muscles during gym ball exercises is thought to increase the stability of the spine and reduce pain and muscle fatigue, resulting in improved joint position sense.

Joint mobilization may be an effective treatment for neurophysiological and mechanical ailments including pain, muscle defense, and muscle spasm and can also can be used effectively to treat joint hypomobility or functionally fixed joints [62]. Gym ball exercises are often used to improve spinal stability and have been widely used in physical therapy for years, and this exercise method is assumed to help reduce the risk of back pain [3]. Gym ball exercises focus on maintaining a neutral spine and are suitable for targeting specific functions of local muscles in the early stages of treatment to improve spinal stability. Trunk muscle endurance can be improved by maintaining a neutral spine position for as long as possible during gym ball exercises, which can reduce the potential for back pain [3]. Gym ball exercises in this study were able to successfully improve the stability and endurance of the spine and reduce pain.

Previous randomized control trials that have included breathing exercises have shown that subjects with an average of one year of moderate chronic back pain showed significant improvement in both pain and functional symptoms after 8 weeks of breathing rehabilitation or physical therapy [26]. Diaphragmatic breathing, progressive muscle relaxation, exercise, self-visualization, and self-hypnosis have been shown to be effective in reducing stress and pain perception [63]. Intervention through relaxation, respiratory reeducation, and other methods is considered to be beneficial in the treatment of chronic pelvic pain [64]. Holloway and West [65] suggested that breathing rehabilitation using the Papworth Method (a series of integrated breathing and relaxation exercises that focus on breathing pattern abnormalities including hyperventilation) can improve the quality of life. In this study, breathing exercises improved various symptoms including lumbar pelvic pain and dysfunction, BPD, and JPE.

There were several limitations of this study. First, it was difficult to control the strength and aerobic exercise exerted by each subject for each task during the experiment. It was also not possible to control other aspects of the subjects' daily environment outside of the rehabilitation center. Further, since the subjects with chronic back pain were recruited from a specific area and institution for this study, the findings of this study cannot be generalized to all individuals with chronic low back pain.

In conclusion, in this study, joint mobilization, gym ball exercises, and breathing exercises improved respiratory pattern abnormalities and joint position sense in persons with chronic lower back pain. Breathing exercises were found to be particularly effective in improving respiratory parameters $\left(\mathrm{ETCO}_{2}, \mathrm{RR}, \mathrm{NQ}\right.$, and Excursion).

\section{Acknowledgements}

The author would like to thank physical therapist, Dr. 
Byung Yun Cho, for his help with the study.

\section{Conflict of Interest}

The authors declared no potential conflicts of interest with respect to the authorship and/or publication of this article.

\section{References}

1. Gatchel RJ, Polatin PB, Mayer TG. The dominant role of psychosocial risk factors in the development of chronic low back pain disability. Spine (Phila Pa 1976) 1995;20:2702-9.

2. Kerr MS, Frank JW, Shannon HS, Norman RW, Wells RP, Neumann WP, et al. Biomechanical and psychosocial risk factors for low back pain at work. Am J Public Health 2001;91:1069-75.

3. Hoogendoorn WE, Bongers PM, de Vet HC, Douwes M, Koes BW, Miedema MC, et al. Flexion and rotation of the trunk and lifting at work are risk factors for low back pain: results of a prospective cohort study. Spine (Phila Pa 1976) 2000;25:3087-92.

4. Marras WS, Granata KP. The development of an EMG-assisted model to assess spine loading during whole-body free-dynamic lifting. J Electromyogr Kinesiol 1997;7:259-68.

5. Adams MA, Freeman BJ, Morrison HP, Nelson IW, Dolan P. Mechanical initiation of intervertebral disc degeneration. Spine (Phila Pa 1976) 2000;25:1625-36.

6. Sluka KA. Pain mechanisms involved in musculoskeletal disorders. J Orthop Sports Phys Ther 1996;24:240-54.

7. Basbaum AI, Bautista DM, Scherrer G, Julius D. Cellular and molecular mechanisms of pain. Cell 2009;139:267-84.

8. CliftonSmith T, Rowley J. Breathing pattern disorders and physiotherapy: inspiration for our profession. Phys Ther Rev 2011;16: 75-86.

9. Kolar P, Sulc J, Kyncl M, Sanda J, Cakrt O, Andel R, et al. Postural function of the diaphragm in persons with and without chronic low back pain. J Orthop Sports Phys Ther 2012;42:352-62.

10. Chaitow L, Bradley D, Gilbert C, Bartley J, Peters D. Recognizing and treating breathing disorders: a multidisciplinary approach. 2nd ed. London: Elsevier Health Sciences UK; 2014.

11. Johnson BD, Saupe KW, Dempsey JA. Mechanical constraints on exercise hyperpnea in endurance athletes. J Appl Physiol (1985) 1992;73:874-86.

12. Hruska RJ Jr. Influences of dysfunctional respiratory mechanics on orofacial pain. Dent Clin North Am 1997;41:211-27.

13. Chaitow L. Breathing pattern disorders, motor control, and low back pain. J Osteop Med 2004;7:33-40.

14. Hodges PW, Eriksson AE, Shirley D, Gandevia SC. Intra-abdominal pressure increases stiffness of the lumbar spine. J Biomech 2005;38:1873-80.

15. Malátová R, Drevikovská $P$. Testing procedures for abdominal muscles using the muscle dynamometer SD02. Proc Inst Mech Eng H 2009;223:1041-8.

16. Claeys K, Brumagne S, Dankaerts W, Kiers H, Janssens L. Decreased variability in postural control strategies in young people with non-specific low back pain is associated with altered proprioceptive reweighting. Eur J Appl Physiol 2011;111:115-23.
17. Martin JH. Modality coding in the somatic sensory system. In: Kandel E, Schwartz J, Jessel T, editors. Principles of neural science. 3rd ed. Norwalk: Appleton \& Lange; 1991.

18. Yahia L, Rhalmi S, Newman N, Isler M. Sensory innervation of human thoracolumbar fascia. an immunohistochemical study. Acta Orthop Scand 1992;63:195-7.

19. Treleaven J. Sensorimotor disturbances in neck disorders affecting postural stability, head and eye movement control. Man Ther 2008;13:2-11.

20. Heikkilä H, Aström PG. Cervicocephalic kinesthetic sensibility in patients with whiplash injury. Scand J Rehabil Med 1996;28: 133-8.

21. Bradley H, Esformes J. Breathing pattern disorders and functional movement. Int J Sports Phys Ther 2014;9:28-39.

22. Yang D, Park S, Kang J, Kim J, Jung D, Kim Y, et al. The effect of spine mobilization technique on balance and the low back pain disability index of patients with chronic back pain. J Korean Soc Integr Med 2018;6:139-48.

23. Lee D, Lee Y. Effects of the trunk stabilization exercise combine in the musical tempo on lumbar lordosis angle, muscle activity and pain. J Korean Soc Integr Med 2018;6:83-9.

24. Park SS, Choi BR. Effects of lumbar stabilization exercises on the flexion-relaxation phenomenon of the erector spinae. J Phys Ther Sci 2016;28:1709-11.

25. Janssens L, McConnell AK, Pijnenburg M, Claeys K, Goossens $\mathrm{N}$, Lysens R, et al. Inspiratory muscle training affects proprioceptive use and low back pain. Med Sci Sports Exerc 2015;47: 12-9.

26. Mehling WE, Hamel KA, Acree M, Byl N, Hecht FM. Randomized, controlled trial of breath therapy for patients with chronic low-back pain. Altern Ther Health Med 2005;11:44-52.

27. Bezzoli E, Andreotti D, Pianta L, Mascheroni M, Piccinno L, Puricelli L, et al. Motor control exercises of the lumbar-pelvic region improve respiratory function in obese men. a pilot study. Disabil Rehabil 2018;40:152-8.

28. Chung S, Lee J, Yoon J. Effects of stabilization exercise using a ball on mutifidus cross-sectional area in patients with chronic low back pain. J Sports Sci Med 2013;12:533-41.

29. Sremakaew M, Jull G, Treleaven J, Barbero M, Falla D, Uthaikhup S. Effects of local treatment with and without sensorimotor and balance exercise in individuals with neck pain: protocol for a randomized controlled trial. BMC Musculoskelet Disord 2018; 19:48.

30. Anderson BE, Bliven $\mathrm{KCH}$. The use of breathing exercises in the treatment of chronic, nonspecific low back pain. J Sport Rehabil 2017;26:452-8.

31. Massé-Alarie, H., Beaulieu, L. D., Preuss, R., \& Schneider, C. (2016). Influence of chronic low back pain and fear of movement on the activation of the transversely oriented abdominal muscles during forward bending. Journal of Electromyography and Kinesiology, 27, 87-94.

32. Laffey JG, Kavanagh BP. Hypocapnia. N Engl J Med 2002;347: 43-53.

33. Courtney R, Greenwood KM, Cohen M. Relationships between measures of dysfunctional breathing in a population with concerns about their breathing. J Bodyw Mov Ther 2011;15:24-34.

34. Chaitow L, Bradley D, Gilbert C. Multidisciplinary approaches to breathing pattern disorders. Edinburgh: Churchill Livingstone; 
2002.

35. Courtney R, van Dixhoorn J, Cohen M. Evaluation of breathing pattern: comparison of a Manual Assessment of Respiratory Motion (MARM) and respiratory induction plethysmography. Appl Psychophysiol Biofeedback 2008;33:91-100.

36. Ellis MJ, Leddy JJ, Willer B. Physiological, vestibulo-ocular and cervicogenic post-concussion disorders: an evidence-based classification system with directions for treatment. Brain Inj 2015; 29:238-48.

37. Burke S, Lynch K, Moghul Z, Young C, Saviola K, Schenk R. The reliability of the cervical relocation test on people with and without a history of neck pain. J Man Manip Ther 2016;24:2104.

38. Bockenhauer SE, Chen H, Julliard KN, Weedon J. Measuring thoracic excursion: reliability of the cloth tape measure technique. J Am Osteopath Assoc 2007;107:191-6.

39. Cahalin LP, Braga M, Matsuo Y, Hernandez ED. Efficacy of diaphragmatic breathing in persons with chronic obstructive pulmonary disease: a review of the literature. J Cardiopulm Rehabil 2002;22:7-21.

40. Kaltenborn FM, Evjenth O, Kaltenborn TB, Vollowitz E. The spine: basic evaluation and mobilization techniques. 2nd ed. Oslo: Olaf Norlis; 1993.

41. Yoon JG. Considerations in Kaltenborn-Evjenth concept related history of orthopedic physical therapy. J Korean Phys Ther Sci 2000;7:275-84.

42. Marshall P, Murphy B. Changes in the flexion relaxation response following an exercise intervention. Spine (Phila $\mathrm{Pa}$ 1976) 2006;31:E877-83.

43. Cho B, Yoon J. Relationship between breathing pattern disorder and joint position sense in patients with chronic low back pain. $\mathrm{J}$ Korean Soc Integr Med 2019;7:1-10.

44. Valenza MC, Cabrera-Martos I, Torres-Sánchez I, Garcés-García A, Mateos-Toset S, Valenza-Demet G. The effects of doming of the diaphragm in subjects with short-hamstring syndrome: a randomized controlled trial. J Sport Rehabil 2015;24:342-8.

45. McLaughlin L. Breathing evaluation and retraining in manual therapy. J Bodyw Mov Ther 2009;13:276-82.

46. Gardner WN. The pathophysiology of hyperventilation disorders. Chest 1996;109:516-34.

47. Garssen B, de Ruiter C, Van Dyck R. Breathing retraining: a rational placebo? Clin Psychol Rev 1992;12:141-53.

48. Hodges PW, Heijnen I, Gandevia SC. Postural activity of the diaphragm is reduced in humans when respiratory demand increases. J Physiol 2001;537(Pt 3):999-1008.

49. Hodges PW, Moseley GL. Pain and motor control of the lumbopelvic region: effect and possible mechanisms. J Electromyogr Kinesiol 2003;13:361-70.
50. Thomas M, McKinley RK, Freeman E, Foy C, Prodger P, Price D. Breathing retraining for dysfunctional breathing in asthma: a randomised controlled trial. Thorax 2003;58:110-5.

51. de Groot EP. Breathing abnormalities in children with breathlessness. Paediatr Respir Rev 2011;12:83-7.

52. Warburton CJ, Jack S. Can you diagnose hyperventilation? Chronic Respir Dis 2006;3:113-5.

53. Stark J, Stark R. The carbon dioxide syndrome : learn why changing your breathing can improve your health and wellbeing. Auckland: Buteyko On Line; 2002.

54. Van Dixhoorn J, Duivenvoorden HJ. Efficacy of Nijmegen questionnaire in recognition of the hyperventilation syndrome. $\mathrm{J}$ Psychosom Res 1985;29:199-206.

55. Annoni JM, Ackermann D, Kesselring J. Respiratory function in chronic hemiplegia. Int Disabil Stud 1990;12:78-80.

56. Röijezon U, Clark NC, Treleaven J. Proprioception in musculoskeletal rehabilitation. part 1: basic science and principles of assessment and clinical interventions. Man Ther 2015;20:36877.

57. Johansson H, Arendt-Nielsen L, Bergenheim M, Blair S, van Dieen J, Djupsjöbacka M, et al. Epilogue: an integrated model for chronic work-related myalgia "Brussels Model". In: Johansson H, Windhorst U, Djupsjöbacka M, Passatore M, editors. Chronic work-related myalgia : neuromuscular mechanisms behind workrelated chronic muscle pain syndromes. Gävle: Gävle University Press; 2003.

58. Godges JJ, Mattson-Bell M, Thorpe D, Shah D. The immediate effects of soft tissue mobilization with proprioceptive neuromuscular facilitation on glenohumeral external rotation and overhead reach. J Orthop Sports Phys Ther 2003;33:713-8.

59. Santilli V, Beghi E, Finucci S. Chiropractic manipulation in the treatment of acute back pain and sciatica with disc protrusion: a randomized double-blind clinical trial of active and simulated spinal manipulations. Spine J 2006;6:131-7.

60. Dutton M. Manual therapy of the spine : an integrated approach. New York: McGraw-Hill Medical Pub; 2002.

61. Norris CM. Back stability. Champaign: Human Kinetics; 2000.

62. Kisner C, Colby LA. Therapeutic exercise : foundations and techniques. 4th ed. Philadelphia: F.A. Davis Company. 2002.

63. Whitmore KE. Complementary and alternative therapies as treatment approaches for interstitial cystitis. Rev Urol 2002;4 Suppl 1:S28-35.

64. Anderson RU, Wise D, Sawyer T, Chan C. Integration of myofascial trigger point release and paradoxical relaxation training treatment of chronic pelvic pain in men. J Urol 2005;174:155-60.

65. Holloway EA, West RJ. Integrated breathing and relaxation training (the Papworth method) for adults with asthma in primary care: a randomised controlled trial. Thorax 2007;62:1039-42. 